\title{
ON THE POSSIBILITY OF LAMINAR FLOW CONTROL ON A SWEPT WING BY MEANS OF PLASMA ACTUATORS
}

\author{
S. L. Chernyshev, A. P. Kuryachii, S. V. Manuilovich, \\ D. A. Rusyanov, and V. V. Skvortsov \\ Central Aerohydrodynamic Institute (TsAGI) \\ 1 Zhukovsky Str., Zhukovsky, Moscow Region 140180, Russia
}

\begin{abstract}
Theoretical assessment of the possibility of laminar flow control (LFC) on a swept wing owing to volumetric force and heat impact of plasma actuators is presented. The proposed approach includes numerical modeling of dielectric barrier discharge (DBD) actuators, calculation of inviscid flow over an infinite span swept wing, calculation of compressible boundary layer spatially modulated in spanwise direction, and numerical solution of linear stability problem for stationary modes of cross-flow-type disturbances. Calculations have been performed for one set of geometrical and physical parameters describing plasma actuators to estimate qualitative features of volumetric force and heat input distributions. Inviscid flow and boundary layer calculations were executed at free stream parameters corresponding to typical cruise flight conditions. Estimation of volumetric force impact necessary for noticeable influence on cross-flowtype instability is obtained.
\end{abstract}

\section{INTRODUCTION}

Development of energy-saving and environmentally appropriate technologies in civil aviation remains one of the industries major objectives [1]. Laminarturbulent transition delay in boundary layer on airplane wing is one of the effective methods of friction drag reduction and, consequently, fuel consumption and atmospheric pollution decrease. The cross-flow-type instability is, as a rule, the main reason of laminar-turbulent transition on a swept wing of modern civil airplanes [2]. Therefore, any method of suppression of this instability would be a key to solve the problem of a swept wing drag reduction, if this method is energy acceptable. The concept of LFC method proposed at TsAGI [3] and based on an attenuation of the cross-flow-type instability due to electrogasdynamic

This is an Open Access article distributed under the terms of the Creative Commons Attribution License 4.0, which permits unrestricted use, distribution, and reproduction in any medium, provided the original work is properly cited. 


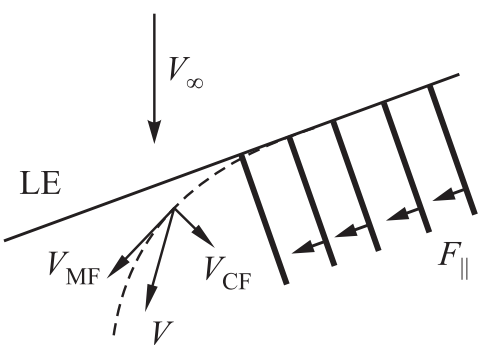

(a)

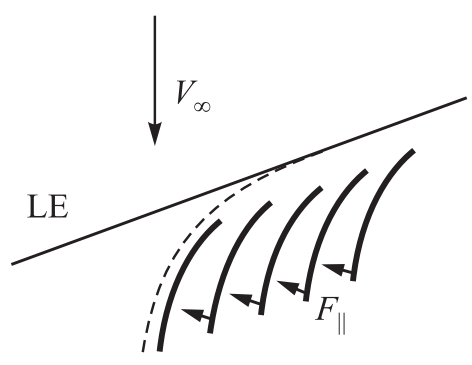

(b)

Figure 1 The concept of EGD LFC on a swept wing

(EGD) force impact on three-dimensional (3D) boundary layer in the vicinity of a swept wing leading edge is illustrated in Fig. 1. Here, $V_{\infty}$ is the free-stream velocity vector; LE denotes the swept wing leading edge; $V$ is the gas velocity vector at some point inside a boundary layer; $V_{\mathrm{MF}}$ and $V_{\mathrm{CF}}$ are the main-flow and cross-flow components of the velocity vector; and dashed curve shows the external inviscid streamline. Plasma actuators operating on the base of near surface DBD seem to be very convenient for this method realization. Solid lines in Fig. 1 show the exposed electrodes of DBD-actuators; $F_{\|}$is the vector component of the volumetric force generated by every actuator and directed parallel to a wing surface.

The simplest configuration of DBD-actuator system is shown in Fig. $1 a$. The actuators are placed continuously both on lower and upper surfaces of a wing perpendicular to its leading edge. Volumetric force impact directed along the leading edge will produce a gas velocity component which will decrease $V_{\mathrm{CF}}$ at some distance from a critical line. The configuration shown in Fig. $1 b$ is more geometrically complex but seems to be more effective and less energy consuming. The curvilinear actuators placed along the external streamline will generate the volumetric force $F_{\|}$directly against $V_{\mathrm{CF}}$. In this case, the DBDactuators may be placed beginning from the line of the cross-flow-type instability origin. In both cases, an attenuation of the cross-flow velocity $V_{\mathrm{CF}}$ results in a decrease of increments of spatial growth of the cross-flow-type instability [4].

Experimental study of the considered LFC method in wind tunnels is very labor-consuming and expensive because of numerous geometric and physical parameters governing this method. Moreover, it is difficult to reproduce cruise flight conditions, namely, transonic Mach number, high Reynolds number, low turbulence level, low static pressure, etc. in wind tunnels. Because of these reasons, a preliminary numerical simulation of EGD LFC method seems to be relevant. The present work is devoted to a theoretical research including a so- 
lution of the following interrelated problems. At first, numerical modeling of spatially periodic DBD-actuator system is carried out with the aim to calculate distributions of time averaged volumetric force and heat release. Main features of these distributons are used then in their analytical approximations. These approximations are necessary for future parametric research because numerical modeling of DBD is very time-consuming [5].

The next step of the present study includes calculation of inviscid flow over infinite span swept wing at typical cruise flight conditions. Then, the calculations of 3D compressible boundary layer spatially periodic along a wing span are executed using the results of the above inviscid calculation and at given spatial distributions of volumetric force and heat release which are used as source terms in momentum and energy equations. Finally, the linear stability of the boundary layer with respect to stationary cross-flow-type instability modes is considered.

Development and optimization of DBD multiactuator system spatially periodic along a wing leading edge and ensuring necessary force impact on large part of a wing surface along a whole leading edge is a substantial problem for practical realization of EGD LFC method [6]. Last years, several tens articles devoted to plasma actuators and their aerodynamic applications are published annually [7-9]. Numerous numerical simulations of various complexity extent, as a rule, deal with a single actuator. The most detailed experimental investigations are executed with a single actuator too. Experimental optimization of multiactuator system is more complex as compared to a single actuator because of a presence of additional parameters governing a system operation [10]. The optimal design of DBD-actuator system must ensure a discharge ignition only at one side of every exposed electrode and exclude or appreciably attenuate it at the other side. Moreover, the mutual interaction between the adjacent actuators worsens characteristics of each actuator [11]. To diminish this damaging interaction, some advanced designs of multiactuator system have been proposed recently [12-14]. Experiments confirmed the effectiveness of the design with additional buried screening electrodes electrically linked with the exposed ones [12] or with additional exposed electrodes under floating potential [14].

\section{NUMERICAL SIMULATION OF DIELECTRIC BARRIER DISCHARGE ACTUATOR SYSTEM}

The considered below design of DBD-actuators in series uses the concept of additional screening buried electrodes but somewhat differs from the original design [12] and takes into account its assumed application on airplane wing. This scheme is shown in Fig. $2 a$. Here, $A$ is the wing skin which must be electricity- 


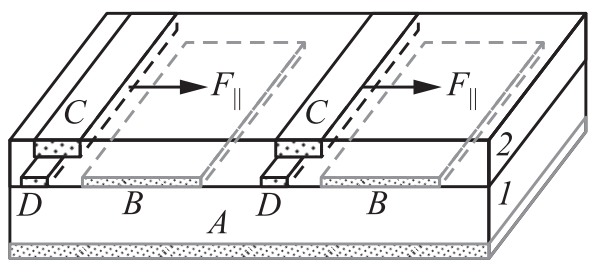

(a)

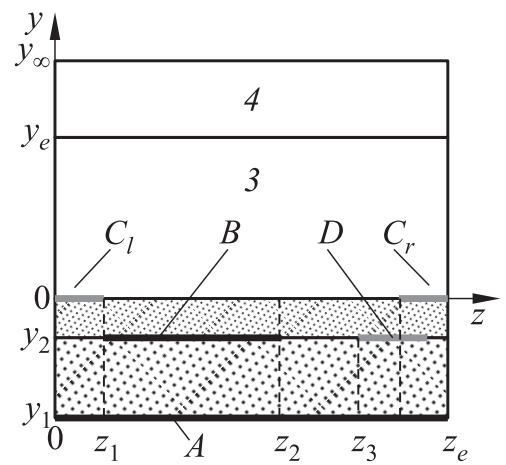

(b)

Figure 2 Design of DBD multiactuator system $(a)$ and calculation domain (b)

conductive because of electrostatic safety requirements, the electric potential of the skin is taken as zero, $B$ are the buried accelerating electrodes under zero potential too, $C$ are the flush-mounted air-exposed electrodes under an alternating electric potential, and the additional buried electrodes $D$ are electrically linked with the exposed ones. All electrodes are separated by dielectric layers 1 and 2 . If the amplitude of the alternating potential is large enough, DBD ignites in the vicinity of the right (active) edges of each exposed electrode $C$. The volumetric electrostatic force arises in these regions due to charge separation in discharge gap caused by significant difference in mobility of electrons and ions. The timeaveraged horizontal component $F_{\|}$of this force is directed from left to right and accelerates a boundary layer in this direction. The additional electrodes $D$ permit to attenuate drastically the electric field strength near the left (passive) edges of the exposed electrodes, thereby excluding or attenuating the discharge in these regions.

The simplest variant of the actuators arrangement shown in Fig. $1 a$ is simulated below because of simplicity of boundary layer calculations. Cartesian coordinates $(x, y, z)$ are introduced with the origin placed on a critical line, the $x$-axis is directed along a wing surface perpendicular to a leading edge, the $y$-axis is directed perpendicular to a wing surface, and the $z$-axis is directed along a leading edge. It means that the electrodes $B, C$, and $D$ are placed along the $x$-axis and the force $F_{\|}$is directed along the $z$-axis. One spatial period of the multiactuator system is considered.

The calculation domain is shown in Fig. $2 b$ and includes subdomains $1-4$. Here, $A$ is the wing skin; $C_{l}$ is the half of some (left) exposed electrode, and $C_{r}$ is the half of the next (right) exposed electrode. The thickness of the skin $A$ and the insulated electrodes $B$ and $D$ is taken to be zero, the thickness of the 
exposed electrodes is equal to $50 \mu \mathrm{m}$. The subdomains 1 and 2 are the dielectric layers, the subdomain 3 corresponds to the discharge gap, and the subdomain 4 is introduced to satisfy the condition of zero potential at infinity [6].

Numerical modeling of DBD in the considered two-dimensional (2D) configuration is executed in the framework of the following statement. The electric potential $\varphi$ is determined by Laplace's equation in the dielectric layers 1, 2, and in the external subdomain 4 (subscripts 1,2, and 4), and by Poisson's equation in the discharge gap (subscript 3 ). The volumetric concentrations of positive ions $n_{p}$, negative ions $n_{n}$, and electrons $n_{e}$ are determined by continuity equations with the use of the drift-diffusion approximation for fluxes of charged particles without taking into consideration convective transfer. So, the mentioned variables are governed by the following equation system:

$$
\begin{gathered}
\Delta \varphi_{i}=0(i=1,2,4) ; \quad \varepsilon_{0} \Delta \varphi_{3}=-e\left(n_{p}-n_{n}-n_{e}\right) ; \quad \mathbf{E}=-\nabla \varphi ; \\
\left.\begin{array}{c}
\frac{\partial n_{p}}{\partial t}+\nabla \boldsymbol{\Gamma}_{p}=k_{i} N n_{e}-k_{\mathrm{dr}} n_{p} n_{e}-k_{r} n_{p} n_{n} ; \\
\frac{\partial n_{n}}{\partial t}+\nabla \boldsymbol{\Gamma}_{n}=0.21 k_{\mathrm{at}} N n_{e}-k_{r} n_{p} n_{n} ; \\
\frac{\partial n_{e}}{\partial t}+\nabla \boldsymbol{\Gamma}_{e}=k_{i} N n_{e}-0.21 k_{\mathrm{at}} N n_{e}-k_{\mathrm{dr}} n_{p} n_{e} ;
\end{array}\right\} \\
\boldsymbol{\Gamma}_{l}=g_{l} b_{l} n_{l} \mathbf{E}-D_{l} \nabla n_{l}(l=p, n, e), \quad g_{p}=1, \quad g_{n}=g_{e}=-1 ; \\
k_{i}=0.79 k_{i \mathrm{~N}}+0.21 k_{i} \mathrm{O}, \quad \lg k_{i \mathrm{~N}}=8.09-\frac{40.29}{\gamma}, \quad \lg k_{i \mathrm{O}}=8.31-\frac{28.57}{\gamma} ; \\
k_{\mathrm{dr}}=2 \cdot 10^{-7}\left(\frac{300}{T_{e}}\right)^{0.7} ; \quad k_{r}=2 \cdot 10^{-6}\left(\frac{300}{T_{p}}\right)^{1.5} ; \lg k_{a t}=10.21-\frac{5.7}{\gamma}
\end{gathered}
$$

Here, $e$ is the elementary charge; $\varepsilon_{0}$ is the dielectric permittivity of vacuum; $t$ is the time; $N\left[\mathrm{~cm}^{-3}\right]$ is the volumetric concentration of air molecules; $k_{i}$ is the ionization rate coefficient taking into account an origin of positive ions of nitrogen and oxygen; $k_{i \mathrm{~N}}$ and $k_{i} \mathrm{O}$ are the individual coefficients of nitrogen and oxygen ionization; $k_{\mathrm{dr}}$ is the rate coefficient of the recombination of electrons with positive ions; $k_{r}$ is the rate coefficient of the ion-ion recombination; $k_{\text {at }}$ is the rate coefficient of dissociative attachment of electrons to oxygen; $\Gamma$ is the drift-diffusion flux; $b$ and $D$ are the coefficients of mobility and diffusion; $\gamma=10^{16} E / N\left[\mathrm{~V} \cdot \mathrm{cm}^{2}\right]$ is the reduced field function; $E[\mathrm{~V} / \mathrm{cm}]$ is the absolute value of the electric field strength; and $T_{e}$ and $T_{p}$ are the temperatures of electrons and ions. The reactions rate coefficients are measured in $\mathrm{cm}^{3} / \mathrm{s}$. Note that the process of the electron detachment from negative ions also takes place in discharge, but its intensity is very small in the considered case of low-temperature 
discharge [15]. Therefore, this reaction is neglected in the present consideration.

The mobility coefficients of ions are defined as

$$
b_{p, n}=C_{p, n} \frac{N_{0}}{N}\left(\frac{300}{T_{p, n}}\right)^{0.3}\left[\mathrm{~cm}^{2} /(\mathrm{V} \cdot \mathrm{s})\right]
$$

where $C_{p}=2.1, C_{n}=3.2$, and $N_{0}=2.69 \cdot 10^{19} \mathrm{~cm}^{-3}$. A distinction of ions $T_{p, n}$ and neutrals $T$ temperatures is neglected. The diffusion coefficients $D_{l}$ $(l=p, n, e)$ are calculated on the base of the Einstein relation using the mobility coefficients. The coefficient of the electron mobility $b_{e}$ and the electron temperature $T_{e}$ in the used locally equilibrium approximation are determined as some given functions of the reduced field $\gamma[16]$.

The periodicity conditions on the left and right boundaries of the calculation domain are used. The boundary conditions on the exposed electrodes take into account secondary electron emission and decelerating influence of the electric field on charged particles. The boundary conditions on the open dielectric surface take into account a precipitation of charged particles, secondary electron emission, finite rates of recombination of charged particles, and electron desorption. These conditions are discussed in detail and presented in $[6,16]$. The electric potential of the exposed and screening electrodes is determined as $\varphi_{C}=-\varphi_{0} \sin (2 \pi f t)$ where $\varphi_{0}$ and $f$ are the amplitude and the frequency of the applied voltage.

Numerical solution of the equation system (1)-(4) with appropriate initial and boundary conditions permits to estimate spatial distributions of volumetric force and heat release generated in each DBD-actuator. These distributions as well as the total force created by an actuator become quasi-periodic in time after several cycles of the applied voltage $[16,17]$. In addition, the discharge characteristics at a given design of actuators and parameters of the applied voltage are sensitive to air pressure because the rate coefficients of ionization and electron attachment depend strongly on the reduced field. Evidently, a streamwise variation of static pressure on a wing surface will influence discharge characteristics. In turn, the numerical modeling of DBD during one cycle of the applied voltage is very time-consuming. Therefore, estimations of main DBD characteristics are obtained below on the base of calculations carried out for one set of the task parameters and the second cycle of the applied voltage [18].

The spatial period of the multiactuator system and the half-width of the exposed electrodes are taken as $z_{e}=10 \mathrm{~mm}$ and $z_{1}=1 \mathrm{~mm}$, correspondingly. Other sizes of the calculation domain are the following (see Fig. 2): $z_{2}=6$, $z_{3}=8, y_{1}=-3, y_{2}=-1, y_{e}=5$, and $y_{\infty}=7 \mathrm{~mm}$. The following physical parameters were used in calculations: the pressure and the temperature of air $p=2.6 \cdot 10^{4} \mathrm{~Pa}$ and $T=223 \mathrm{~K}$, the amplitude and the frequency of the applied harmonic voltage $\varphi_{0}=5 \mathrm{kV}$ and $f=10 \mathrm{kHz}$, the dielectric con- 


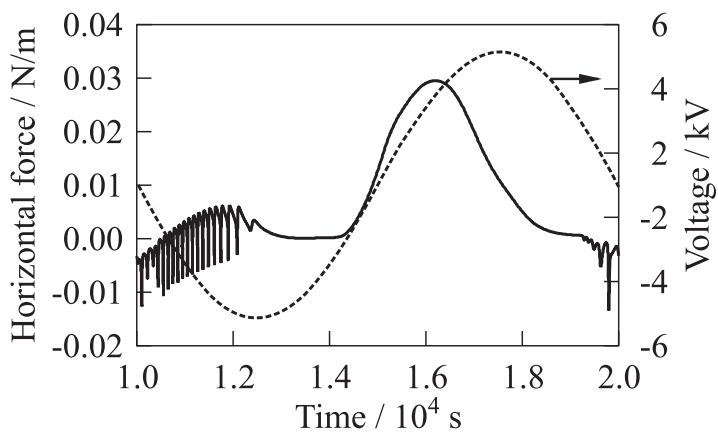

(a)

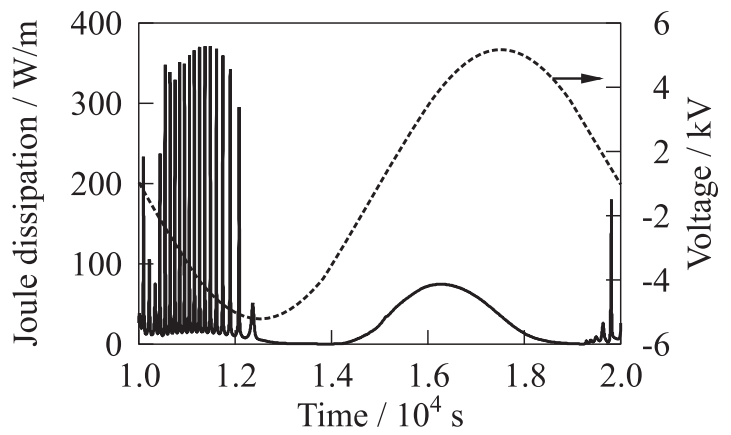

(b)

Figure 3 Spatial integrated horizontal force $(a)$ and Joule dissipation $(b)$ during the second cycle of DBD

stants of the insulation layers $\varepsilon_{1}=2.1$ (Teflon) and $\varepsilon_{2}=3.5$ (Kapton), the coefficients of the secondary ion-electron emission on dielectric and electrode surfaces equal 0.05 , the frequency of electron desorption from dielectric surface equals $5 \mathrm{kHz}$ [19], and the electron-ion recombination coefficient on dielectric surface equals $10^{-7} \mathrm{~cm}^{2} / \mathrm{s}$ [19].

The time dependencies of horizontal force and Joule dissipation integrated on the area of the discharge gap $S_{3}$ during the second cycle of the applied voltage are shown in Fig. 3. These functions are calculated according to the following formulas:

$$
F_{\|}(t)=\int_{S_{3}} e E_{z}\left(n_{p}-n_{n}-n_{e}\right) d z d y ; \quad J(t)=\int_{S_{3}} e \mathbf{E}\left(\boldsymbol{\Gamma}_{p}-\boldsymbol{\Gamma}_{n}-\boldsymbol{\Gamma}_{e}\right) d z d y
$$

Dashed curves in Fig. 3 represent the potential of the exposed and screening electrodes. 
First of all, note that the discharge ignition on the right exposed electrode $C_{r}$ (see Fig. 2) is absent at the task parameters specified above. In turn, an increase of the applied voltage amplitude up to $6 \mathrm{kV}$ in additional calculations resulted in a discharge ignition on $C_{r}$.

The physical explanation of the presented behavior of the horizontal force and Joule dissipation is given in [18]. The main characteristics of DBD-actuator such as the total average horizontal force $\left\langle F_{\|}\right\rangle$and Joule dissipation $\langle J\rangle$ per unit length of the exposed electrode and the energy efficiency coefficient Eff $\equiv\left\langle F_{\|}\right\rangle /\langle J\rangle[20]$ have been calculated using (5) according to

$$
\left.\begin{array}{c}
\left\langle F_{\|}\right\rangle=\frac{1}{\Delta t} \int_{t}^{t+\Delta t} F_{\|} d t=6.74 \cdot 10^{-3} \mathrm{~N} / \mathrm{m} ; \\
\langle J\rangle=\frac{1}{\Delta t} \int_{t}^{t+\Delta t} J d t=2.51 \mathrm{~W} / \mathrm{m} ; \\
\mathrm{Eff}=2.69 \cdot 10^{-4} \mathrm{~s} / \mathrm{m} ; \quad \Delta t=10^{-4} \mathrm{~s} .
\end{array}\right\}
$$

For the aim of further boundary layer calculations with force and heat impact of DBD-actuators, it is impotant to consider some features of heat transfer in DBD. The point is that the preliminary theoretical estimations revealed significant influence of the volumetric heat input on boundary layer flow and its stability characteristics at a presence of negative streamwise pressure gradient [21]. Not all Joule dissipation $\langle J\rangle$ is spent on gas heating in discharge gap because of gas flow presence. Some part of the electron energy comes firstly in the energy of vibrational degrees of freadom of air molecules. Then, this vibrational energy relaxes into translational degrees of freadom during a finite time. A gas flow can influence a region of this relaxation and, hence, heat release. The time of the vibrational-translational relaxation $\tau_{\mathrm{VT}}$ for nitrogen and oxygen molecules depends on gas pressure $p$ and temperature $T$ according to the following expressions [22]:

$$
\left.\begin{array}{l}
p \tau_{\mathrm{VT}}^{\left(\mathrm{N}_{2}\right)}=6.5 \cdot 10^{-4} \exp \left(\frac{137}{T^{1 / 3}}\right)[\mathrm{Pa} \cdot \mathrm{s}] ; \\
\left.p \tau_{\mathrm{VT}}^{\left(\mathrm{O}_{2}\right)}=2.7 \cdot 10^{-3} \exp \left(\frac{90}{T^{1 / 3}}\right)[\mathrm{Pa} \cdot \mathrm{s}] \quad(T<500 \mathrm{~K}) \cdot\right\}
\end{array}\right\}
$$

These dependences are presented in Fig. 4 for two values of gas temperature. Vertical solid line marks the pressure value used in the present calculations. According to $(7), \tau_{\mathrm{VT}}=162 \mathrm{~s}$ for nitrogen and $\tau_{\mathrm{VT}}=0.29 \mathrm{~s}$ for oxygen at $p=2.6 \cdot 10^{4} \mathrm{~Pa}$ and $T=223 \mathrm{~K}$.

The fractions of the electron power channeled into vibrational exitation in air depending on the reduced field function at mentioned pressure and typical for DBD relative electron concentration $n_{e} / N=10^{-9}$ have been calculated on the 


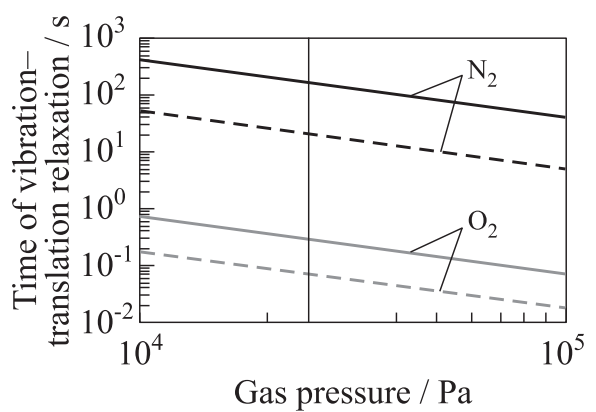

Figure 4 Vibration-translation relaxation time: solid lines refer to $223 \mathrm{~K}$ and dashed lines to $300 \mathrm{~K}$

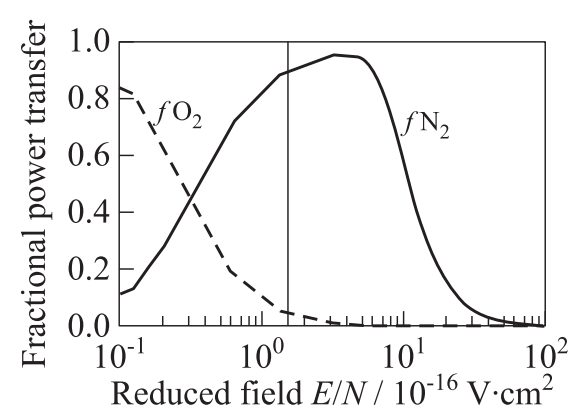

Figure 5 Vibrational fractions of power transfer

base of two-term approximation to the Boltzmann equation [23] and are shown in Fig. 5. Dashed curve $f \mathrm{O}_{2}$ corresponds to oxygen and the solid curve $f \mathrm{~N}_{2}$ corresponds to nitrogen. The average fractions of the total Joule dissipation channeled into vibrational exitation have been calculated using above functions according to

$$
\left.\begin{array}{l}
\left\langle J_{\mathrm{N} 2}\right\rangle=-\frac{1}{\Delta t} \int_{t}^{t+\Delta t} d t \int_{S_{3}} f \mathrm{~N}_{2} e \mathbf{E} \cdot \boldsymbol{\Gamma}_{e} d z d y \cong 0.24\langle J\rangle ; \\
\left\langle J_{\mathrm{O} 2}\right\rangle=-\frac{1}{\Delta t} \int_{t}^{t+\Delta t} d t \int_{S_{3}} f \mathrm{O}_{2} e \mathbf{E} \cdot \boldsymbol{\Gamma}_{e} d z d y \cong 5 \cdot 10^{-3}\langle J\rangle \cdot
\end{array}\right\}
$$

So, about a fourth of the total Joule dissipation coming into vibrational exitation of nitrogen may fly out of a discharge gap owing to gas flow in the considered particular case. Exitation of oxygen may be neglected. Further relaxation of this power into gas heating essentially depends on gas pressure and temperature according to (7). Note that about $40 \%$ of the total Joule dissipation is generated by the current of positive ions.

The spatial distributions of the time-averaged horizontal force and Joule dissipation have been calculated according to

$$
\left.\begin{array}{l}
F(y, z)=\frac{1}{\Delta t} \int_{t}^{t+\Delta t} e E_{z}\left(n_{p}-n_{n}-n_{e}\right) d t \\
Q(y, z)=\frac{1}{\Delta t} \int_{t}^{t+\Delta t} e \mathbf{E}\left(\boldsymbol{\Gamma}_{p}-\boldsymbol{\Gamma}_{n}-\boldsymbol{\Gamma}_{e}\right) d t, \quad \Delta t=0.1 \mathrm{~ms}
\end{array}\right\}
$$




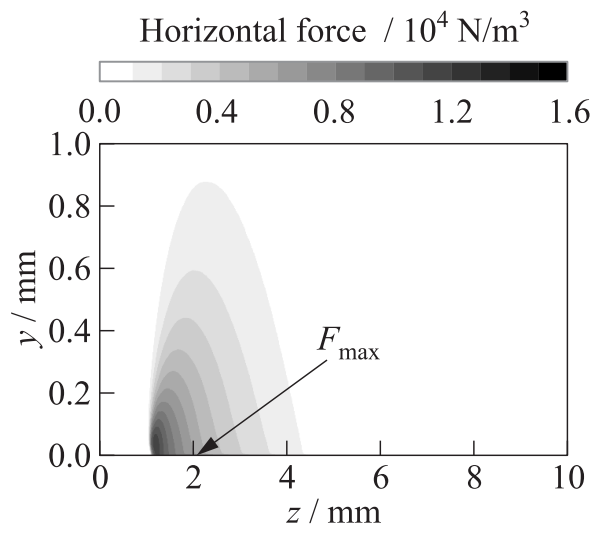

(a)

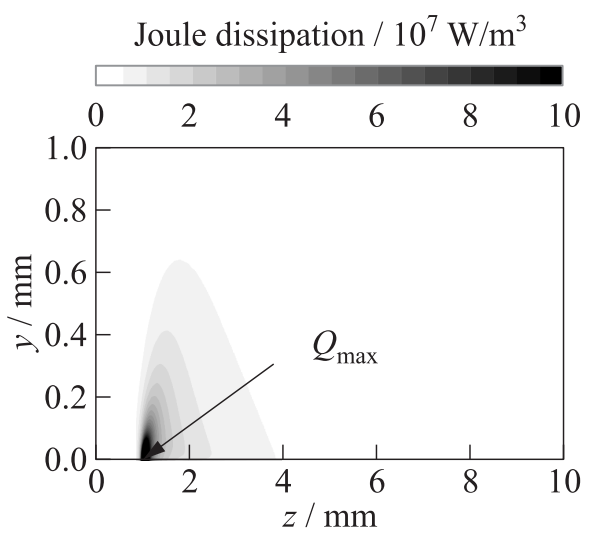

(b)

Figure 6 Time averaged horizontal force $\left(F_{\max }=1.6 \cdot 10^{4} \mathrm{~N} / \mathrm{m}^{3}\right)$ and Joule dissipation $\left(Q_{\max }=2.8 \cdot 10^{9} \mathrm{~W} / \mathrm{m}^{3}\right)$

The distributions (9) created in the discharge gap at given geometric and phycal parameters are shown in Fig. 6. There are two regions of intense horizontal force. The first region is observed near the edge of the exposed electrode $(z=1 \mathrm{~mm})$ and the second very narrow region is distant from the edge up to $z=2 \mathrm{~mm}$ where the maximal value $F_{\max }=1.6 \cdot 10^{4} \mathrm{~N} / \mathrm{m}^{3}$ is reached on dielectric surface. The calculated distribution of Joule dissipation is monotonous, very sharp, with maximum $Q_{\max }=2.8 \cdot 10^{9} \mathrm{~W} / \mathrm{m}^{3}$ achieved on the electrode edge where both the electric field strength and the electric current density are extremal. Note that the qualitative behavior of the time-averaged volumetric force presented in Fig. 6 is similar to that obtained in detailed experiments [24].

These distributions have been used in calculations of compressible boundary layer on an infinite span swept wing in [18]. The obtained results have shown that the main characteristics of DBD-actuators determined by the values (6) are insufficient for noticeable influence on cross-flow stability. Moreover, the vertical size of the volumetric force region was too large because the boundary layer thickness was small enough (essentially less $1 \mathrm{~mm}$ ).

As it was mentioned above, a choice of optimal geometric and physical parameters of DBD multiactuator system on the base of its numerical modeling described above requires very time. Therefore, the following simplified approach is proposed to facilitate subsequent parametric numerical investigations of the considered LFC method and to fomulate demands to main parameters of DBDactuator system. It is assumed that the characteristic features of the spatial distributions of the volumetric force and Joule dissipation shown in Fig. 6 can 


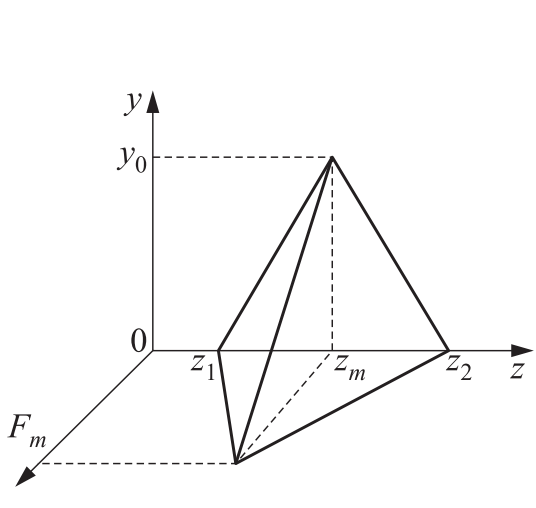

(a)

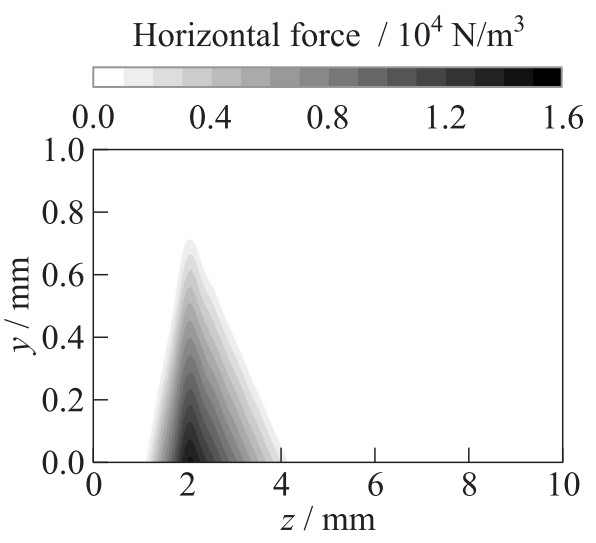

(b)

Figure 7 Analytic approximation of volumetric force and heat sources

be reproduced by analytical approximation in the form of pyramid shown in Fig. 7 according to the following formulas $\left(0 \leq y \leq y_{0}\right)$ :

$$
F= \begin{cases}F_{m}\left(\frac{z-z_{1}}{\delta z}-\frac{y}{y_{0}}\right), & \delta z=z_{m}-z_{1}, \frac{y}{y_{0}} \delta z+z_{1} \leq z \leq z_{m} \\ F_{m}\left(1-\frac{z-z_{m}}{\Delta z}-\frac{y}{y_{0}}\right), & \Delta z=z_{2}-z_{m}, z_{m} \leq z \leq z_{2}-\frac{y}{y_{0}} \Delta z\end{cases}
$$

Predefined values of $\left\langle F_{\|}\right\rangle, y_{0}, z_{1}$, and $z_{2}$ determine the maximal value of this distribution $F_{m}=6\left\langle F_{\|}\right\rangle /\left[y_{0}\left(z_{2}-z_{1}\right)\right]$. Figure 7 demostrates this approximation for the volumteric force shown in Fig. 6 at $\left\langle F_{\|}\right\rangle=6.74 \cdot 10^{-3} \mathrm{~N} / \mathrm{m}, y_{0}=0.8$, $z_{1}=1, z_{m}=2$, and $z_{2}=4.4 \mathrm{~mm}$.

Proposed parametric study implies variations of the average volumetric force $\left\langle F_{\|}\right\rangle$, the energy efficiency coefficent, the distance between actuators $z_{e}$, and the characteristic vertical and horizontal sizes of force and heat sources. This approach is used below to evaluate the value of the average volumetric force $\left\langle F_{\|}\right\rangle$necessary for remarkable attenuation of the cross-flow-type instability on a swept wing for typical cruise flight conditions at fixed geometric parameters of volumetric sources and relation $\left\langle J_{\mathrm{N}_{2}}\right\rangle /\langle J\rangle$ in (8).

\section{BOUNDARY LAYER CALCULATION}

The effect of DBD-actuators on cross-flow-type instability is estimated by the example of a flow over an infinite span swept wing with the sweep angle $\chi=30^{\circ}$. 
The static pressure $p_{\infty}=2.6 \cdot 10^{4} \mathrm{~Pa}$, the air temperature $T_{\infty}=223 \mathrm{~K}$ corresponding to numerical simulation of DBD-actuators in the previous section, and Mach number $\mathrm{M}_{\infty}=0.8$ are taken as the main free stream parameters. They determine other flow parameters necessary for further boundary layer calculations: flow velocity $V_{\infty}=240 \mathrm{~m} / \mathrm{s}$, the air density $\rho_{\infty}=0.41 \mathrm{~kg} / \mathrm{m}^{3}$, and the dynamic viscosity coefficient $\mu_{\infty}=1.33 \cdot 10^{-5} \mathrm{~kg} /(\mathrm{m} \cdot \mathrm{s})$.

The external boundary conditions for calculations of the compressible boundary layer have been obtained from the calculation of 2D inviscid flow over LV6 DLR airfoil at zero angle of attack on the base of the Euler equations. It is supposed that volumetric and force impact of plasma actuators is concentrated entirely inside a boundary layer. The spanwise modulation of the boundary layer displacement thickness resulting from actuators impact and corresponding viscous-inviscid interaction have not been taken into consideration.

The boundary layer flow in the vicinity of a wing leading edge is characterized by Reynolds number $\operatorname{Re}=\rho_{\infty} V_{\infty} l / \mu_{\infty}$ determined by streamwise length $l=V_{\infty} /\left(d u_{e}(0) / d x\right)$, where the $x$-coordinate is directed along a wing surface perpendicular to a leading edge, and $u_{e}$ is the $x$-component of the external velocity obtained from inviscid calculation. The airfoil chord length normal to a leading edge $L$ is related with the characteristic length $l$ by the formula $L=l d u^{\prime}(0) / d x^{\prime}$, where the dimensionless velocity $u_{e}^{\prime}$ and the coordinate $x^{\prime}$ are measured in $V_{\infty} \cos \chi$ and $L$, respectively. According to executed $2 \mathrm{D}$ inviscid flow calculation, $d u_{e}^{\prime}(0) / d x^{\prime}=107.7$. The value $l=0.03 \mathrm{~m}$ was taken in the present calculations; hence, the airfoil chord length equals $L=3.23 \mathrm{~m}$.

Calculated distributions of the nondimensional $x$-component of the external flow velocity $u_{e}^{*}=u_{e} / V_{\infty}$ in the vicinity of the wing leading edge and its streamwise gradient are shown in Fig. 8. Zero angle of attack for a given airfoil

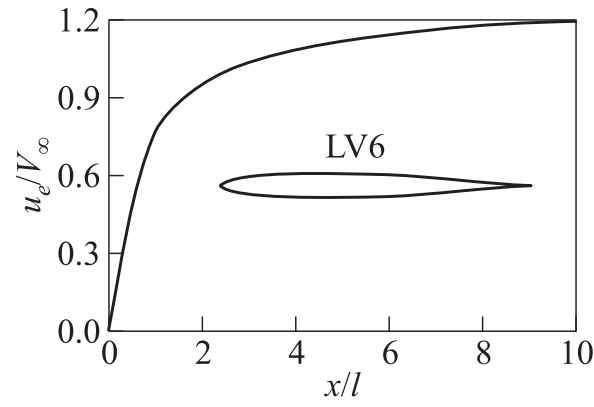

(a)

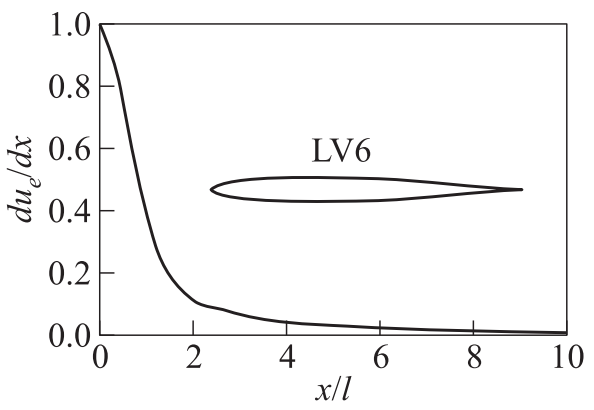

(b)

Figure 8 Dimensionless external streamwise velocity $(a)$ and its streamwise gradient $(b)$ 


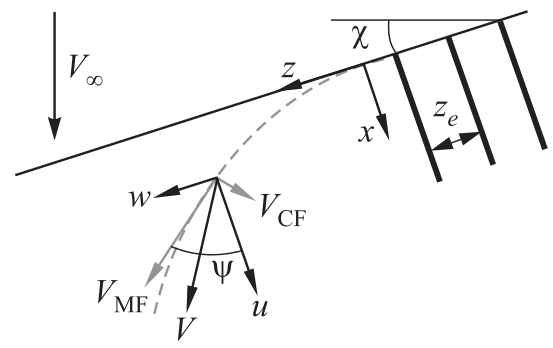

Figure 9 Decomposition of the velocity vector in boundary layer flow

has been taken in inviscid calculation because of a long enough part of streamwise flow acceleration and, as a consequence, intense cross-flow in the boundary layer.

The boundary layer flow is defined by the velocity components $u, v$, and $w$, the static pressure $p$, the air density $\rho$, the static enthalpy $h=c_{p} T$, and the average vibrational energy per unit mass of air $\omega$. It is assumed that the infinite set of DBD-actuators is placed on a wing surface with the step $z_{e}$ in the spanwise direction, as it is shown in Fig. 9. The exposed electrodes of the actuators begin from the critical line on the leading edge and are directed along the $x$-axis. It is supposed that each actuator creates volumetric force and heat input distributions which do not depend on the $x$-coordinate. It is evident that a variation of static pressure along a wing chord will influence discharge characteristics and, hence, distributions of the volumetric force and heat input. But this effect is neglected in the current approximate consideration.

In reality, DBD-actuators generate both horizontal (parallel to a dielectric surface) and vertical (normal to the surface) components of the volumetric force. Theoretical estimations [25] show that the impact of the vertical force component directed to the solid surface on a boundary layer flow can be noticeable if this component is much greater than the horizontal one and is not uniform along the surface. But in reality, the opposite situation takes place, i. e., the horizontal force exceeds essentially the vertical one [24]. Therefore, the influence of the vertical volumetric force on a boundary layer flow is not taken into account.

A possibility to use the usual boundary layer approximation in the considered case is proved in [21]. The mentioned above characteristics of the boundary layer flow are governed by the following system of the equations and boundary conditions:

$$
\frac{\partial(\rho u)}{\partial x}+\frac{\partial(\rho v)}{\partial y}+\frac{\partial(\rho w)}{\partial z}=0 ; \quad p=\frac{\gamma-1}{\gamma} \rho h ;
$$




$$
\begin{aligned}
& \left.\rho u \frac{\partial u}{\partial x}+\rho v \frac{\partial u}{\partial y}+\rho w \frac{\partial u}{\partial z}=-\frac{d p}{d x}+\frac{\partial}{\partial y}\left(\mu \frac{\partial u}{\partial y}\right) ;\right) \\
& \left.\rho u \frac{\partial w}{\partial x}+\rho v \frac{\partial w}{\partial y}+\rho w \frac{\partial w}{\partial z}=F+\frac{\partial}{\partial y}\left(\mu \frac{\partial w}{\partial y}\right) ;\right\} \\
& \rho u \frac{\partial h}{\partial x}+\rho v \frac{\partial h}{\partial y}+\rho w \frac{\partial h}{\partial z} \\
& =u \frac{d p}{d x}+\mu\left[\left(\frac{\partial u}{\partial y}\right)^{2}+\left(\frac{\partial w}{\partial y}\right)^{2}\right]+\frac{\partial}{\partial y}\left(\frac{\mu}{\operatorname{Pr}} \frac{\partial h}{\partial y}\right)+(1-r) Q+\rho \frac{\omega-\omega_{0}}{\tau_{\mathrm{VT}}} \\
& \rho u \frac{\partial \omega}{\partial x}+\rho v \frac{\partial \omega}{\partial y}+\rho w \frac{\partial \omega}{\partial z}=\frac{\partial}{\partial y}\left(\frac{\mu}{\operatorname{Sc}} \frac{\partial \omega}{\partial y}\right)+r Q-\rho \frac{\omega-\omega_{0}}{\tau_{\mathrm{VT}}}
\end{aligned}
$$

where

$$
\left.\begin{array}{c}
\mu(T)=1.47 \cdot 10^{-6} \frac{T^{3 / 2}}{T+114} ; \quad \gamma=1.4 ; \quad \operatorname{Pr}=0.72 ; \quad \text { Sc }=0.9 ; \\
y=0: \quad u=v=w=\frac{\partial h}{\partial y}=\omega=0, ; \\
y=y_{e}: \quad u=u_{e}, w=w_{e}, h=h_{e}, \omega=0
\end{array}\right\}
$$

where

$$
w_{e}=V_{\infty} \sin \chi ; \quad h_{e}=h_{\infty}+0.5\left(V_{\infty}^{2}-u_{e}^{2}-w_{e}^{2}\right) .
$$

Here, $F$ and $Q$ are the distributions of the horizontal volumetric force and total Joule dissipation prescribed in the form (10); $r=\left\langle J_{\mathrm{N} 2}\right\rangle /\langle J\rangle$ is the vibrational fraction of Joule dissipation, the last terms in Eqs. (13) and (14) simulate the process of the vibrational-translational relaxation with characteristic time $\tau_{\mathrm{VT}}$ and the equilibrium value of the vibrational energy $\omega_{0}=0$ for relatively cold gas considered here. The wall boundary condition for enthalpy $h$ (15) implies a thermal insulated wall.

The specified above task parameters determine the value of the Reynolds number $\operatorname{Re}=2.03 \cdot 10^{5}$ and the boundary layer thickness on the critical line $\delta_{0} \approx 5 \mathrm{Re}^{-1 / 2} l \approx 0.33 \mathrm{~mm}$. The following constant parameters determining the volumetric force and heat sources have been used in calculations: the spatial period of the actuator system $z_{e}=5 \mathrm{~mm},\left\langle F_{\|}\right\rangle=0.02 \mathrm{~N} / \mathrm{m}, y_{0}=0.3, z_{1}=0.5$, $z_{m}=1$, and $z_{2}=2.5 \mathrm{~mm}$ for volumetric force source, and $y_{0}=0.2, z_{1}=0.5$, $z_{m}=0.8$, and $z_{2}=1.5 \mathrm{~mm}$ for Joule dissipation source. Two values of the energy efficiency coefficient have been used. The first value is close to the calculated in the previous section $\mathrm{Eff}=2.5 \cdot 10^{-4} \mathrm{~s} / \mathrm{m}$, i. e., the averaged Joule dissipation equals $\langle J\rangle=80 \mathrm{~W} / \mathrm{m}$. The second value is taken twice as large Eff $=5 \cdot 10^{-4} \mathrm{~s} / \mathrm{m}$ and, hence, $\langle J\rangle=40 \mathrm{~W} / \mathrm{m}$. The second value was considered to evaluate a sensitivity of boundary layer flow and its stability to energy efficiency of plasma 


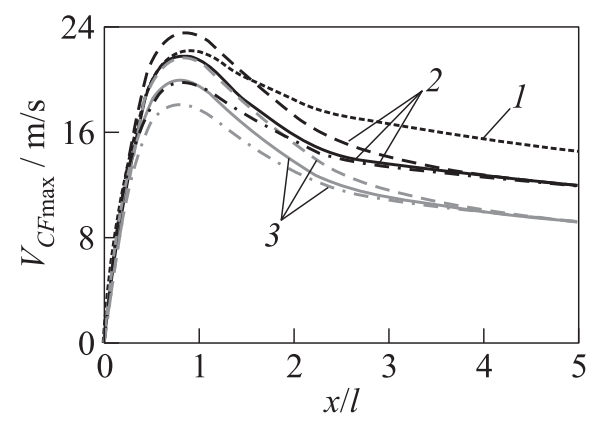

(a)

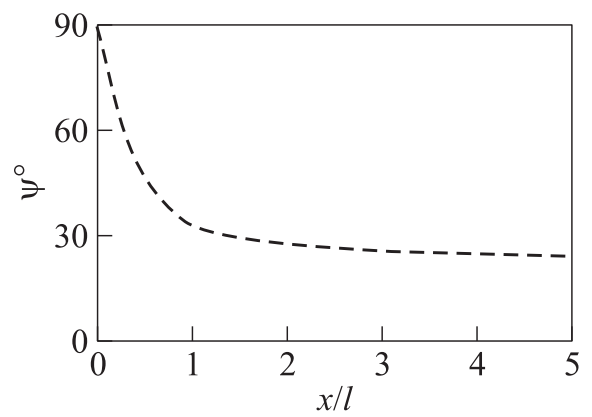

(b)

Figure 10 Maximal cross-flow velocity $(a)$ and external streamline angle $(b)$ vs. dimensionless streamwise distance: 1 - without impact; 2 - impact at Eff $=2.5$ $\times 10^{-4} \mathrm{~s} / \mathrm{m}$; and 3 - impact at Eff $=5 \cdot 10^{-4} \mathrm{~s} / \mathrm{m}$

actuators. The vibrational fraction of Joule dissipation is taken according to (8) $r=0.24$ and the relaxation time equals $\tau_{\mathrm{VT}}=162 \mathrm{~s}$. Note that the last term in the energy Eq. (13) is negligible in the considered case because of very large $\tau_{\mathrm{VT}}$, i. e., it is not necessary to solve the vibrational energy Eq. (14). This need can appear at greater pressure and temperature and less flow velocity, for example, in simulation of wind tunnel experiments.

As the considered boundary layer flow is spanwise periodical, a numerical solution of the system (11)-(15) at given distributions of flow parameters on the external boundary $y_{e}$ has been executed with the use of the expansion of all dependent variables and volumetric source terms (10) in finite Fourier series on the $z$-variable and subsequent solution of the resulting $2 \mathrm{D}$ equation systems.

Figure 10 demonstrates the streamwise distributions of extremal values of the cross-flow velocity $V_{\mathrm{CF}}$ calculated according to (see Fig. 9):

$$
\left.\begin{array}{rlrl}
V_{\mathrm{CF}} & =u \sin \psi-w \cos \psi ; & V_{\mathrm{MF}} & =u \cos \psi+w \sin \psi ; \\
\operatorname{tg} \psi & =\frac{w_{e}}{u_{e}} ; & w_{e} & =V_{\infty} \sin \chi .
\end{array}\right\}
$$

Dotted curve 1 in Fig. $10 a$ shows the maximal cross-flow velocity in the boundary layer without impact of actuators. The dashed curve in Fig. $10 b$ represents the variation of the angle between the $x$-axis and the external inviscid streamline (see Fig. 9). Three curves 2 represent maximal (dashed), minimal (dashdotted), and average (solid) maximums of the cross-flow velocity at force and heat impact of actuators reached in each cross section $y-z$ for the energy efficiency coefficient Eff $=2.5 \cdot 10^{-4} \mathrm{~s} / \mathrm{m}$. Three curves 3 refer to greater energy efficiency coefficient Eff $=5 \cdot 10^{-4} \mathrm{~s} / \mathrm{m}$. Note that average value of any flow function is determined as zero term in the corresponding Fourier series. 

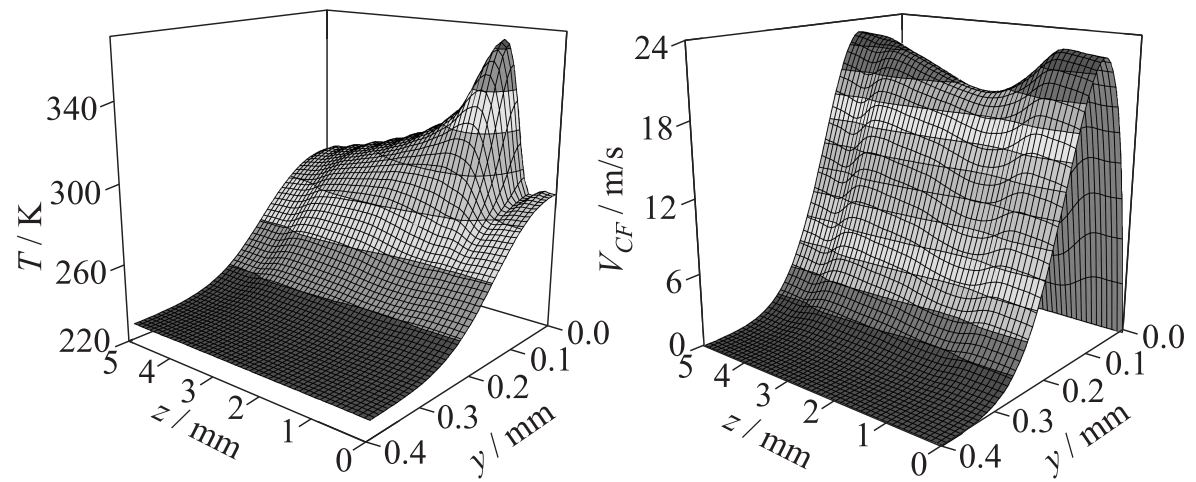

(a)
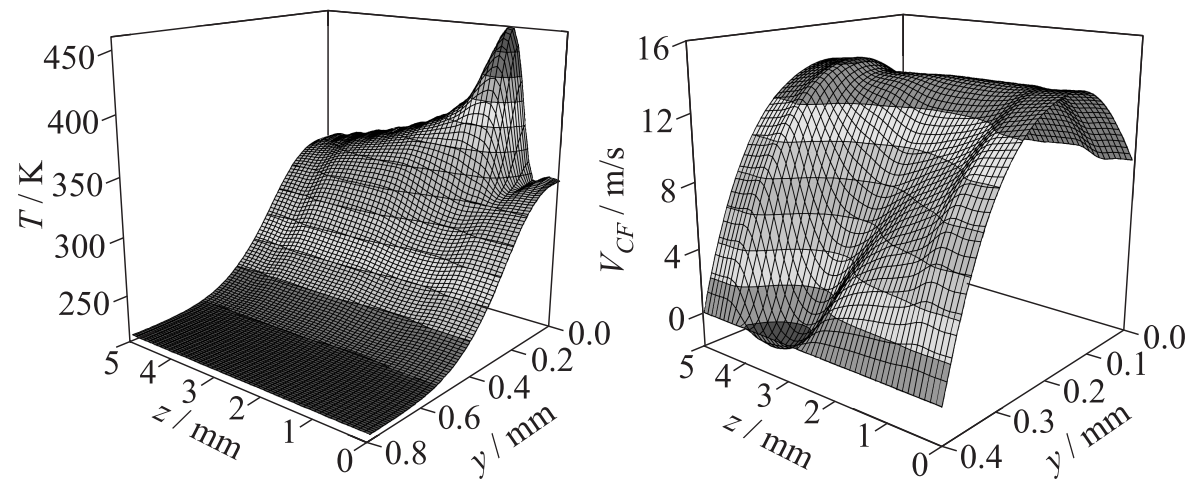

(b)

Figure 11 Distributions of gas temperature and cross-flow velocity in the crosssection $x / l=1(a)$ and $3(b)$

The boundary layer flow becomes highly nonuniform along a span because of force and heat impact of actuators, as it is seen in Fig. 11a where the gas temperature and the cross-flow velocity distributions are shown in the streamwise cross section $x / l=1$ for Eff $=2.5 \cdot 10^{-4} \mathrm{~s} / \mathrm{m}$. The upper (dashed) curve 2 in Fig. 10 lies above the dotted curve 1 up to the cross section $x / l=1.5$ reflecting an accelerating effect of heat input on streamwise velocity component $u$ [21]. This effect results in an increase in the cross-flow velocity $V_{\mathrm{CF}}$ according to (16). The force impact resulting in an increase in $w$-component and, hence, a decrease of $V_{\mathrm{CF}}$ becomes more apparent with a decrease of external streamline angle $\psi$ according to (16) and Fig. 10.

The amplitude of the spanwise modulation of the cross-flow velocity decays gradually downstream despite the fact that the heat impact increases, according 
to Fig. $11 b$ where the temperature distribution in whole boundary layer and the cross-flow velocity distribution in near-wall region are shown in the cross section $x / l=3$ for the same Eff $=2.5 \cdot 10^{-4} \mathrm{~s} / \mathrm{m}$. Moreover, negative cross-flow velocity appears near the wall owing to a force impact. Extremal negative value of the cross-flow velocity varies from $-2 \mathrm{~m} / \mathrm{s}$ at $x / l=3$ down to $-4.5 \mathrm{~m} / \mathrm{s}$ at $x / l=5$. At that, the maximal temperature achieving at dielectric surface increases up to $530 \mathrm{~K}$ at $x / l=5$. An increase in energy efficiency of DBD-actuators noticeably weakens negative influence of heat input on cross-flow velocity. Curves 3 in Fig. 10 demonstrate that a force impact of actuators prevails above a heat impact in all considered part of boundary layer flow at Eff $=5 \cdot 10^{-4} \mathrm{~s} / \mathrm{m}$. At that extremal negative value of the cross-flow velocity in the cross section $x / l=5$ varies not many up to $-4.8 \mathrm{~m} / \mathrm{s}$ but at the same time, the maximal temperature decreases remarkably down to $380 \mathrm{~K}$.

One can conclude that the considered impact on boundary layer flow is not optimal and seems to be too strong. At least, an intense heating of a dielectric surface can result in its destruction and deterioration of DBD-actuator operational characteristics [26].

\section{CALCULATIONS OF CROSS-FLOW STABILITY}

The influence of plasma actuators impact on boundary layer stability is estimated in the framework of the linear stability equation system of Dunn-Lin [27]. The spatial modulation of the boundary layer flow along a span demonstrated in Fig. 11 is not taken into account in the present simplified consideration. It means that only zero terms of Fourier expansions of undisturbed flow functions are used in the present stability analysis. Note that the influence of the spanwise flow modulation on stability of 3D compressible boundary layer was estimated in [28] on the base of Floquet method. It was shown that this modulation results in some decrease of increments of spatial growth of the cross-flow-type modes. But the possibility of appearance of other instability modes in spanwise modulated flows demands additional investigation.

Only stationary cross-flow-type disturbances are considered which are characterized by the angle between the external streamline and the wave vector direction in the range $85^{\circ}-90^{\circ}$. The well-known $e^{N}$ method is used to estimate the position of laminar-turbulent transition [2]. So, the disturbances of all flow functions and the $N$ factor are defined as follows:

$$
\begin{aligned}
q(x, y, z)=q^{*}(y) \exp [i(\alpha x+\beta z)] & =q^{*}(y) \exp \left(-\alpha_{i} x\right) \exp \left[i\left(\alpha_{r} x+\beta z\right)\right] ; \\
N(x) & =-\int_{x_{0}}^{x} \alpha_{i} d x .
\end{aligned}
$$


Here, $q^{*}$ is the complex eigenfunction; $\alpha=\alpha_{r}+i \alpha_{i}$ is the complex eigenvalue; $\alpha_{r}$ and $\beta$ represent the wavenumber components in the $x$ and $z$ directions; $\alpha_{i}$ represents increment of spatial growth $\left(\alpha_{i}<0\right)$ or decrement of decay $\left(\alpha_{i}>0\right)$ of disturbances; and $x_{0}$ is the initial coordinate where $\alpha_{i}$ becomes negative.

The so-called fixed $\beta$ strategy [2] is used for $N$ factor computation, that is, the streamwise distributions of the eigenvalue $\alpha$ and the $N$ factor are calculated for a set of various fixed spanwise wavenumbers $\beta$. Figure 12 demostrates calculated distributions of dimensionless increments of spatial growth $\alpha^{\prime}=\alpha l \mathrm{Re}^{-1 / 2}$ and $N$ factors in streamwise direction for several dimensionless spanwise wavenumbers $\beta^{\prime}=\beta l \operatorname{Re}^{-1 / 2}$.

The $e^{N}$ method implies that a laminar-turbulent transition occurs when any $N$ factor reaches a some predefined value $N_{T}$. The cross-flow induced transition is characterized by $N_{T}=8-10$ for the fixed $\beta$ strategy used here [2]. Using the lower value $N_{T}=8$, one can see in Fig. $12 a$ that transition can occur in usual boundary layer without impact of actuators at the distance $x / l=3.3$ for $\beta^{\prime}=0.3$. Impact of actuators even at low energy efficiency coefficient Eff $=2.5 \cdot 10^{-4} \mathrm{~s} / \mathrm{m}$ $(\langle J\rangle=40 \mathrm{~W} / \mathrm{m})$ prevents transition at least in the considered part of the boundary layer. An increase of energy efficiency of actuators up to two times ensures more significant reserve of flow stability, as the lower Fig. 12 demonstrates. The legends in Figs. $12 b$ and $12 c$ indicate the average densities of the volumetric force and the electric power per unit of a wing surface calculated as $F_{S}=\left\langle F_{\|}\right\rangle / z_{e}$ and $J_{S}=\langle J\rangle / z_{e}$, respectively.

The comparison of Figs. $12 a$ and $12 b$ (left column) representing spatial growth increments and also the curves 1 and 2 in Fig. 10 representing maximums of the average cross-flow velocity shows that noticeable effect of plasma actuators at Eff $=2.5 \cdot 10^{-4} \mathrm{~s} / \mathrm{m}$ appears at the distance $x / l>1$ where the external streamline angle drops down to about $30^{\circ}$. The distance of the instability origin is estimated as $x_{0} / l=0.21-0.26$ for the considered range of the spanwise wavenumbers. One can suppose that a significant strengthening of the actuators influence on cross-flow stability may be reached by the use of curvilinear electrodes of actuators oriented along the external streamline begining from the distance $x_{0} / l \approx 0.2$.

The average densities of the volumetric force and the electric power per unit of a wing surface in the considered case are estimated as $F_{S}=4 \mathrm{~N} / \mathrm{m}^{2}$ and $J_{S}=16 \mathrm{~kW} / \mathrm{m}^{2}$ at the energy efficiency coefficient Eff $=2.5 \cdot 10^{-4} \mathrm{~s} / \mathrm{m}$. This power about twice as large than the mechanical power required to overcome the turbulent skin friction on a wing in cruise flight. However, DBD-actuators required to remove a laminar-turbulent transition caused by cross-flow-type instability may cover only a few percent of total wing surface. Therefore, significant savings in mechanical power may be obtained due to laminarization about a half of a wing surface (approximately, up to static pressure minimum) if the transition induced by Tollmien-Schlichting instability will be suppressed, for example, due to favourable streamwise pressure gradient. 

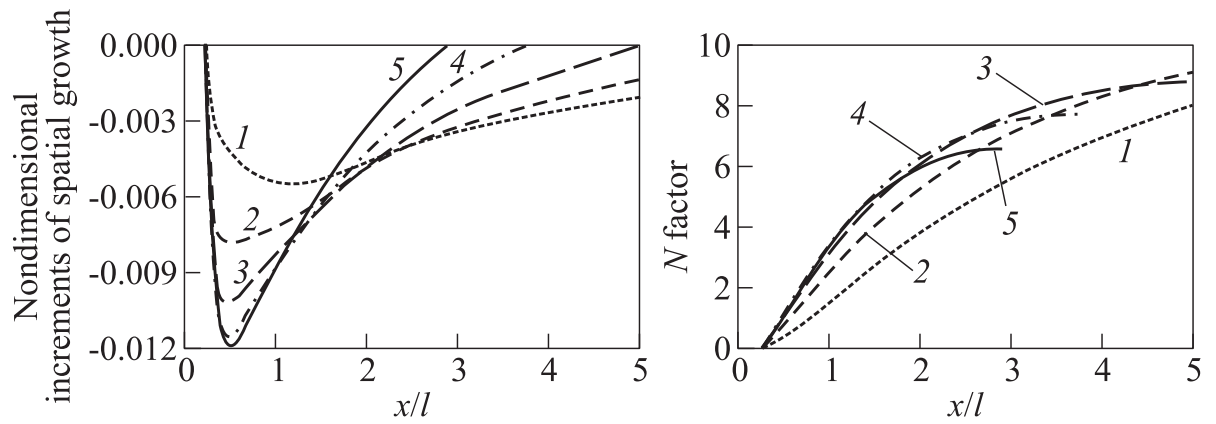

(a)
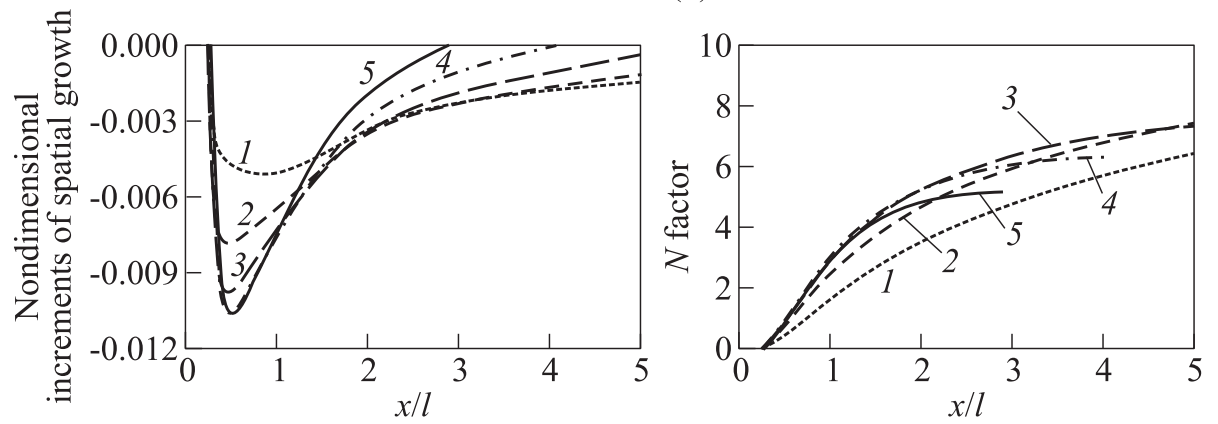

(b)
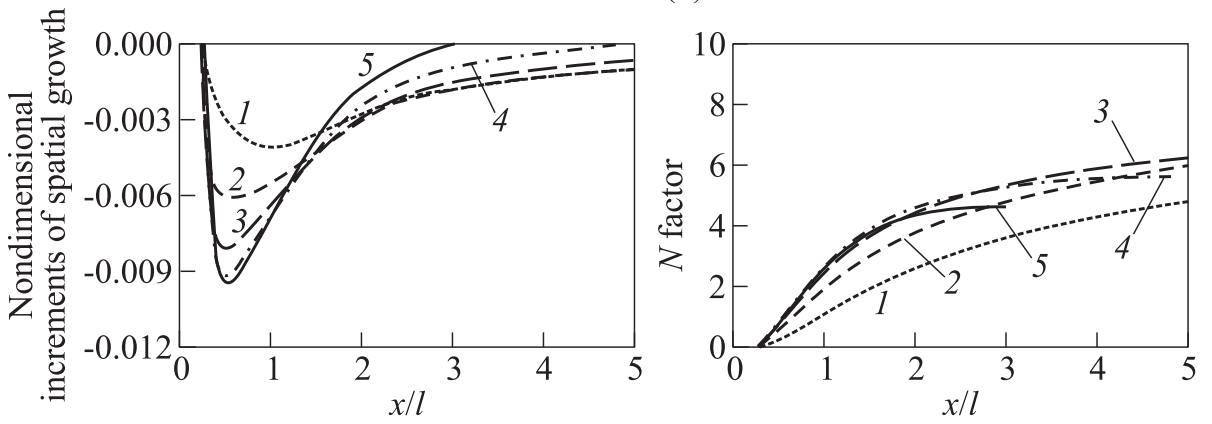

(c)

Figure 12 Streamwise distributions of nondimensional increments of spatial growth and $N$ factor: (a) no impact; $(b) F_{s}=4 \mathrm{~N} / \mathrm{m}^{2}$ and $J_{s}=16 \mathrm{~kW} / \mathrm{m}^{2}$; and $(c) F_{s}$ $=4 \mathrm{~N} / \mathrm{m}^{2}$ and $J_{s}=8 \mathrm{~kW} / \mathrm{m}^{2}$. Nondimensional spanwise wavenumber: $1-0.2 ; 2-$ $0.25 ; 3-0.3 ; 4-0.35$; and $5-0.4$ 


\section{CONCLUDING REMARKS}

The proposed theoretical approach seems to be pertinent for preliminary evaluations of EGD LFC method and its parametric study. The average density of the volumetric force per unit of a wing surface of several $\mathrm{N} / \mathrm{m}^{2}$ seems to be sufficient for delay the laminar-turbulent transition induced by cross-flow instability at cruise flight conditions. But further optimization of the considered method demands a consideration of curvilinear actuators oriented along the external streamline. The influence of strong spanwise nonuniformity of boundary layer flow caused by volumetric force and heat sources on cross-flow stability must be also studied.

Both the boundary layer flow and the cross-flow stability characteristics are very sensitive to volumetric heat input and, hence, to energy efficiency of plasma actuators. It demands thorough optimization of DBD-actuator system including its miniaturization.

\section{ACKNOWLEDGMENTS}

The research was supported by Russian Foundation for Basic Research (project No. 12-01-00086).

\section{REFERENCES}

1. Abbas, A, J. de Vicente, and E. Valero. 2013. Aerodynamic technologies to improve aircraft performance. Aerosp. Sci. Technol. 28:100-132.

2. Arnal, D., and G. Casalis. 2000. Laminar-turbulent transition prediction in threedimensional flows. Prog. Aerosp. Sci. 36:173-191.

3. Chernyshev, S. L., A. Ph. Kiselev, and A.P. Kuryachii. 2011. Laminar flow control research at TsAGI: Past and present. Prog. Aerosp. Sci. 47:169-185.

4. Mack, L. M. 1979. On the stability of the boundary layer on a transonic swept wing. AIAA Paper No. 1979-264.

5. Soloviev, V.R. 2012. Analytical estimation of the thrust generated by a surface dielectric barrier discharge. J. Phys. D Appl. Phys. 45:025205. 12 p.

6. Kuryachii, A.P., D. A. Rusyanov, S.L. Chernyshev, and V.V. Skvortsov. 2013. About increase of efficiency of plasma multi-actuator system for boundary layer control. TsAGI Sci. J. 44:305-326.

7. Moreau, T. 2007. Airflow control by non-thermal plasma actuators. J. Phys. D Appl. Phys. 40:605-636.

8. Corke, T. C., C. L. Enloe, and S.P. Wilkinson. 2010. Dielectric barrier discharge plasma actuators for flow control. Annu. Rev. Fluid Mech. 42:505-529. 
9. Wang, J. J., K.-S. Choi, L. Feng, T. Jukes, and R. Whalley. 2013. Recent developments in DBD plasma flow control. Prog. Aerosp. Sci. 62:52-78.

10. Thomas, F. O., T. C. Corke, M. Iqbal, A. Kozlov, and D. Schatzman. 2009. Optimization of dielectric barrier discharge plasma actuators for active aerodynamic flow control. AIAA J. 47:2169-2178.

11. Do, H., W. Kim, M.A. Capelli, and M. G. Mungal. 2009. Cross-talk in multiple dielectric barrier discharge actuators. Appl. Phys. Lett. 92:071504.

12. Benard, N., J. Jolibois, A. Mizuno, and E. Moreau. 2009. Innovative three-electrode design for definition of multiple dielectric barrier discharge actuators. 2009 Electrostatic Joint Conference Proceedings. Paper No. 1-17.

13. Erfani, R., C. Hale, and K. Kontis. 2011. The influence of electrode configuration and dielectric temperature on plasma actuator performance. AIAA Paper No. 2011955.

14. Berendt, A., J. Podlinski, and J. Mizeraczyk. 2012. Multi-DBD actuator with floating inter-electrode for aerodynamic control. Nukleonika 57:249-252.

15. Kossyi, I. A., A. Yu. Kostinsky, A. A. Matveyev, and V.P. Silakov. 1992. Kinetic scheme of the non-equilibrium discharge in nitrogen-oxygen mixtures. Plasma Sources Sci. Technol. 1:207-220.

16. Kuryachii, A. P., D. A. Rusyanov, and V. V. Skvortsov. 2011. Features of numerical modeling of a dielectric barrier discharge. TsAGI Sci. J. 42:37-52.

17. Enloe, C. L., G. I. Font, T. E. McLaughlin, and D. Orlov. 2008. Surface potential and longitudinal electric field measurements in the aerodynamic plasma actuator. AIAA J. 46:2730-2740.

18. Chernyshev, S. L., A.P. Kuryachii, S. V. Manuilovich, D. A. Rusyanov, and V.V. Skvortsov. 2013. Attenuation of cross-flow-type instability in compressible boundary layer by means of plasma actuators. AIAA Paper No. 2013-321.

19. Golubovskii, Yu. B., V. A. Maiorov, J. Behnke, and J. F. Behnke. 2002. Influence of interaction between charged particles and dielectric surface over a homogeneous barrier discharge in nitrogen. J. Phys. D Appl. Phys. 35:751-761.

20. Porter, C., J. Baughn, T. McLaughlin, C. Enloe, and G. Font. 2006. Temporal force measurements on an aerodynamic plasma actuator. AIAA Paper No. 2006-104.

21. Kuryachii, A.P., and S. V. Manuilovich. 2011. Attenuation of cross-flow-type instability in a 3D boundary layer due to volumetric force impact. TsAGI Sci. J. 42:345-360.

22. Mnatsatanyan, A.H., and G. V. Naigis. 1985. Balance of the vibrational energy in discharges in air. High Temp. 23:640-648.

23. Hagelaar, G. J. M, and L. C. Pitchford. 2005. Solving the Boltzmann equation to obtain electron transport coefficients and rate coefficients for fluid models. Plasma Sources Sci. Technol. 14:722-733.

24. Benard, N., A. Debien, and E. Moreau. 2013. Time-dependent volume force produced by a non-thermal plasma actuator from experimental velocity field. J. Phys. D Appl. Phys. 46:245201. 12 p.

25. Kuryachii, A. P. 2006. Effect of a space-time source structure simulating a dielectric barrier discharge on the laminar boundary layer. Fluid Dyn. 41:366-374. 
26. Hanson, R., J. Kimelman, and P. Lavoie. 2013. Effect of dielectric degradation on dielectric barrier discharge plasma actuator performance. AIAA Paper No. 2013397.

27. Dunn, D. W., and C. C. Lin. 1952. The stability of the laminar boundary layer in a compressible fluid for the case of three-dimensional disturbances. J. Aeronaut. Sci. 19:491-502.

28. Manuilovich, S. V., and E. S. Asmolov. 2013. Effect of spanwise periodicity of the force and thermal impact on gas flow stability in tree-dimensional boundary layer. Models and methods of aerodynamics. 13th School-Seminar (International) Proceedings. Moscow, Russia. 145-146. [In Russian.] 